\title{
Pruritus bei atopischer Dermatitis: Mechanismen und Therapie
}

\section{Pruritus in Atopic Dermatitis: Mechanisms and Therapy}

\section{Autor}

Institut

\section{S. Ständer}

Abteilung Klinische Neurodermatologie, Klinik und Poliklinik für Hautkrankheiten, Universitätsklinikum Münster

\section{Bibliografie}

Dol $10.1055 / \mathrm{s}-2007-966210$

Akt Dermatol 2007; 33;

131-134 @ Georg Thieme

Verlag KG Stuttgart · New York ISSN 0340-2541

Korrespondenzadresse

PD Dr. Sonja Ständer

Abteilung für klinische

Neurodermatologie

Klinik und Poliklinik für

Hautkrankheiten

Universitätsklinikum Münster

Von-Esmarchstr. 58

48149 Münster

sonja.staender@uni-

muenster.de

\section{Zusammenfassung \\ $\nabla$}

Die Inzidenz der atopischen Dermatitis bei Kindern hat in den letzten Jahren stark zugenommen. Chronischer Pruritus ist hierbei eine schwer therapierbare Begleiterscheinung, der bei Kindern vornehmlich mit verschiedenen Topika zu behandeln ist. Konventionelle Therapien wie topische Antihistaminika sind in den meisten Fällen unwirksam und prolongieren den Pruritus. Dadurch kann der Pruritus weiter chronifizieren, zu sekundären Kratzläsionen führen und die Lebensqualität erheblich verschlechtern. Aktuell stehen neue antipruritische Substanzen zur Verfügung, die in unterschiedliche, pruritogene Me- chanismen eingreifen und auch bei Kindern angewandt werden können. Eingesetzt werden Substanzen, die die kutane Pruritusentstehung blockieren bzw. die Weiterleitung von Pruritus an das zentrale Nervensystem hemmen. Wichtig ist auch eine Durchbrechung peripherer Sensibilisierungsprozesse. Leider stehen bei Kindern derzeit keine zugelassenen Substanzen zur Verfügung, die die Weiterleitung von Pruritus im zentralen Nervensystem oder die zentrale Sensibilisierung unterbinden. Wichtig ist es daher, bei Kindern möglichst früh Pruritus zu behandeln, um Sensibilisierungs- und Chronifizierungsprozessen vorzubeugen.

\section{Einleitung}

\section{$\nabla$}

Die Inzidenz der atopischen Dermatitis hat in den letzten Jahren stark zugenommen [1]. Die Erkrankung beginnt bei ca. einem Drittel der Patienten bereits mit dem 3. Lebensmonat; der Großteil ist vor dem 5. Lebensjahr betroffen. Neben den Erythemen korreliert die Ausbreitung und Intensität des Pruritus mit dem Leidensdruck verbunden mit einer Einschränkung der Lebensqualität und erfordert daher eine schnell wirksame und effiziente antipruritische Therapie. Gerade bei Kindern, denen in sehr jungem Alter die Erkrankung und der Pruritus sowie die Notwendigkeit topischer Basistherapien nicht im vollen Umfang erklärt werden kann, ist eine rasche Linderung des Pruritus unerlässlich [2]. Akuter und chronischer Pruritus ist ein Kardinalsymptom der atopischen Dermatitis. Akuter Pruritus entsteht im Rahmen einer akuten Exazerbation der Neurodermitis und ist überwiegend den entzündlichen Mechanismen zu attributieren. Chronischer Pruritus, der auch im Intervall bestehen bleiben kann, beruht auf komplexen Mechanismen wie der Sensibilisierung von Neurorezep- toren oder Aussprossung von Nervenfasern [2,4]. Daraus resultiert u.a. die Alloknesis, d.h. eine Juckempfindung, die durch nicht-pruritogene Reize ausgelöst wird, wie z.B. Pruritus nach mechanischen Stimuli. Konventionelle Therapien durchbrechen diese Mechanismen nicht und neue Substanzen sind zur therapeutischen Intervention notwendig, deren Entwicklung in den letzten Jahren durch das Verständnis der Neuromechanismen erleichtert wurde [5]. In dieser Arbeit sollen einige dieser Mechanismen und die daraus resultierenden Therapieoptionen dargestellt werden.

\section{Nervenfasern und Neurorezeptoren $\nabla$}

Die Haut ist mit einem dichten Netzwerk von Nervenfasern ausgestattet. Diese lassen sich in A-Fasern (schnell-leitende (20-120 m/s), myelinisierte Nervenfasern für schnellen Schmerz und mechanische Stimuli) und C-Fasern (langsamleitende, $(0,5-1 \mathrm{~m} / \mathrm{s})$ unmyelinisierte, sog. polymodale sensorische Nervenfasern für Brennschmerz, Hitze, Kälte, Pruritus) unterscheiden 


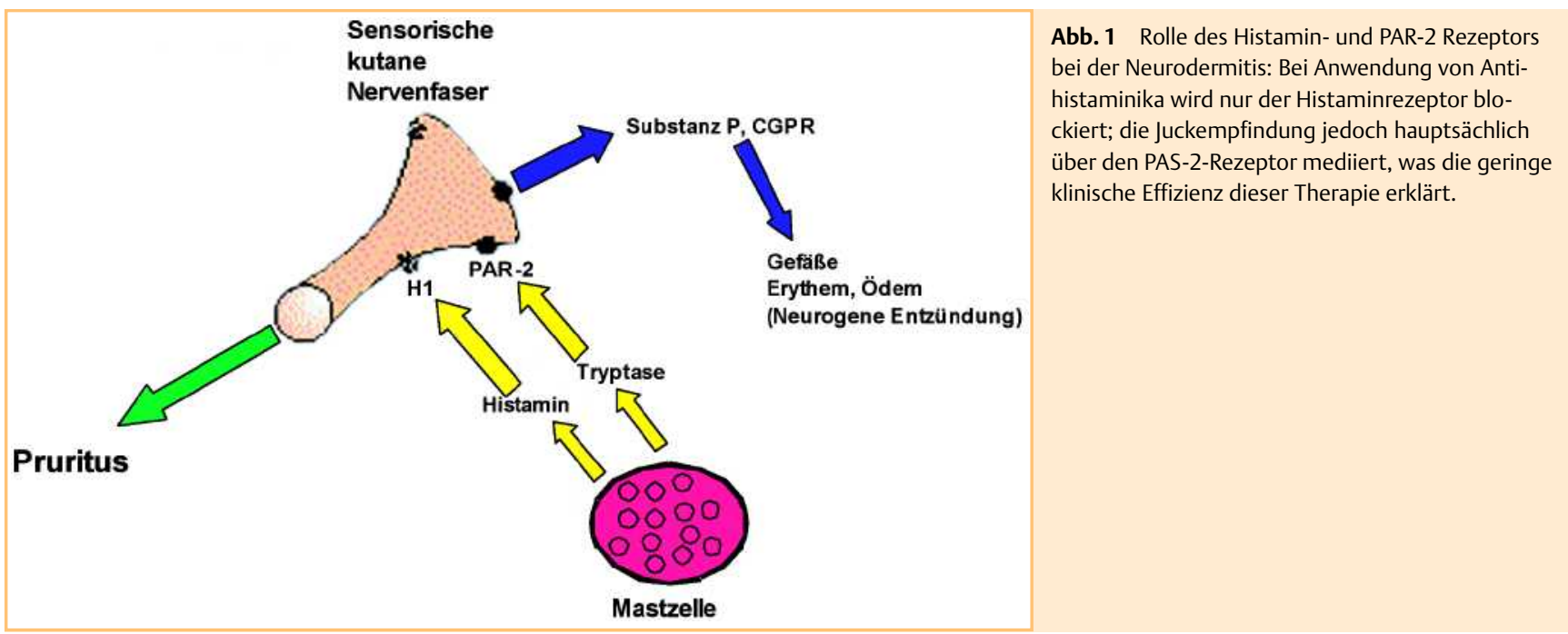

[4]. Die C-Fasern enden als freie Nervenendigungen an der Epidermis-Dermisgrenze bzw. in der Epidermis und sind mit einer großen Anzahl an Neurorezeptoren ausgestattet. Die sensorischen Nervenfasern mit einer großen Anzahl an Neuropeptiden üben einerseits afferente Funktionen aus. Andererseits werden die Neuropeptide bei der Aktivierung der Nervenfaser ausgeschüttet und nehmen eine Reihe von efferenten Funktionen an kutanen Zielstrukturen wahr. So wirken Substanz P(SP) und Calcitonin gene-related Peptide (CGRP) nach Freisetzung aus den sensorischen Fasern auf die Gefäße und verursachen eine Gefäßdilatation und Plasmaextravasation; es kann ein klinisch relevantes Erythem und Ödem entstehen („neurogene Entzündung“). Des Weiteren wurde als Einfluss dieser Faktoren eine vermehrte Keratinozytenproliferation und -differenzierung beschrieben [6]. Neuropeptide wirken darüber hinaus auch auf kutane Mastzellen. Vielfach wurde die Freisetzung von MastzellMediatoren durch SP beschrieben, die jedoch von anderen Autoren bestritten und mit Mikrodialyse-Verfahren nicht bestätigt werden konnte [7]. Eine Vielzahl der Mediatoren aus den Mastzellen wirkt wiederum pruritogen, wie z.B. Histamin, Tryptase, Endothelin oder verschiedene Interleukine. Dies trägt zu einer Perpetuierung der Juckempfindung bei. Interessanterweise wurde bei Patienten mit AD eine Vermehrung der kutanen sensorischen Nervenfasern sowie eine Vermehrung der Neuropeptide wie SP und CGRP nachgewiesen [8-10]. Diese Befunde scheinen damit zumindest teilweise den chronischen Pruritus zu erklären.

Der bekannteste Neurorezeptor ist der Histamin-1-Rezeptor. Lange galt Histamin als einziger Mediator, der direkt an dem Rezeptor auf den Nerven Pruritus auslösen kann; ein Dogma, was seit ca. 10 Jahren widerlegt ist. Ebenfalls im Gegensatz zu früheren Annahmen steht die Erkenntnis, dass Histamin bei der atopischen Dermatitis von nur untergeordneter Bedeutung ist. Patienten mit atopischer Dermatitis sind für experimentellen, Histamin-induzierten Pruritus deutlich weniger empfindlich als Hautgesunde [11]. Dies würde u.a. auch die nur geringe Effizienz von Antihistaminika bei der Neurodermitis erklären [12,13]. Bewährt hat sich jedoch der Einsatz z.T. sedierender Antihistaminika, die auch schon bei Kindern angewandt werden können, z. B. Cetirizin Tropfen oder Sirup ab dem 1. Lebensjahr [2]. Vor fast einem Jahrzehnt wurde auf den sensorischen C-Nervenfasern der Proteinase-aktivierte Rezeptor 2 (PAR-2) nachgewiesen
[14]. Eine Aktivierung mit Tryptase führt zu einer neurogenen Entzündung und klinischen Pruritusempfindung. In weiteren Untersuchungen konnte gezeigt werden, dass Patienten mit atopischer Dermatitis einen intensiveren Pruritus im Vergleich zu Hautgesunden nach Aktivierung des Rezeptors empfinden [15]. Diese Befunde ergänzen die Erkenntnisse zum Histaminrezeptor (Histamin: wenig Pruritusempfinden, Tryptase: starker Pruritus) und liefern eine weitere Erklärung für das klinische Versagen von Antihistaminika bei Patienten mit AD ( $\bullet$ Abb. 1). Der Einsatz von PAR-2-Antagonisten erscheint daher eine sinnvolle Therapieergänzung bei Pruritus im Rahmen der atopischen Dermatitis. Momentan sind die bekannten Antagonisten jedoch nur für den experimentellen Einsatz geeignet und die klinische Anwendung einschließlich Phase I Studien steht aus.

\section{TRPV1 und Capsaicintherapie} $\nabla$

Der TRPV1-Rezeptor (früher: Capsaicin-Rezeptor oder VanilloidRezeptor 1/VR1) ist im zentralen und peripheren Nervensystem vorhanden und wird auch auf den kutanen Nervenfasern exprimiert. Seine Besonderheit besteht in der Art des Rezeptors, die sich therapeutisch nutzen lässt: der Rezeptor ist ein Kationenkanal, der sich bei einmaliger Stimulierung kurzfristig öffnet, bei kontinuierlicher Stimulation eine permanente Öffnung („Desensibilisierung“) aufweist. Durch diesen Kationenkanal wird das Aktionspotential der Nervenfaser, was letztendlich zu der Empfindung von Pruritus und Brennschmerz führt, sowie die Ausschüttung der Neuropeptide reguliert. Entsprechend kann bei kontinuierlicher Stimulierung durch eine Weitstellung der Kanäle ein intra- und extrazelluläres Ionengleichgewicht erzielt werden, was die Auslösung eines Aktionspotentials und somit die Auslösung von Brennschmerz und Juckempfindung verhindert [16]. Capsaicin wird daher seit dem 19. Jahrhundert bei Schmerz und Pruritus unterschiedlicher Genese topisch angewandt, z.B. als ABC-Pflaster. Auch bei subakuten und chronischen Formen der Neurodermitis oder auch der Prurigoform der AD hat sich der Einsatz von Capsaicin bewährt. Durch die initiale Auslösung von Brennschmerz empfiehlt sich eine langsame Eindosierung der Capsaicinkonzentration $(0,025 \%-0,05 \%-0,075 \%-0,1 \%)$. Dazu stehen leider derzeit keine kommerziellen Präparate zur Verfügung und es muss auf eine Magistralrezeptur (Rp. 1\% Ex- 
tractum Capsici 2,5 g in Ung. Leniens ad $100 \mathrm{~g}$, entspricht 0,025\% Capsacincreme) verwiesen werden. Bei Kindern ab dem 10. Lebensjahr ist Capsaicin nur in Ausnahmefällen anzuwenden. Durch Kontakt der Creme mit den Händen und versehentliches Einbringen in das Auge kann eine starke Reizung erzielt werden, die bei Kindern unter dem 10. Lebensjahr fast nicht vermieden werden kann [17].

Es besteht ein interessanter Zusammenhang zwischen dem Capsaicinrezeptor und der Therapie mit den Calcineurininhibitoren. Pimecrolimus und Tacrolimus weisen gel. in der Initialphase der Therapie die Symptome einer neurogenen Entzündung auf, wie es auch unter Capsaicinanwendung beobachtet werden kann. Neue experimentelle Untersuchungen am Tiermodell haben gezeigt, dass sowohl Tacrolimus als auch Pimecrolimus an dem Capsaicinrezeptor TRPV1 binden, eine neurogene Entzündung und Mastzelldegranulation auslösen [18,19]. Dies erklärt zum einen das initiale Brennen der Therapie. Zum anderen wird hierdurch die antipruritische Wirkung der Therapie erklärt, die in allen Studien der letzten Jahre dokumentiert wurde. Der Effekt setzt innerhalb der ersten Therapietage ein und steigert sich mit kontinuierlicher Anwendung. Bei verschiedenen Formen der atopischen Dermatitis ist dies somit eine Alternative zu der Capsaicintherapie, die darüber hinaus bei kleinen Kindern Anwendung finden kann und rezeptierbar ist [20-22].

\section{Cannabinoidrezeptoren und Therapie mit Agonisten \\ $\nabla$}

Aktuelle neuroanatomische und neurophysiologische Untersuchungen zeigten, dass das Cannabinoidsystem mit Liganden und Rezeptoren funktionell in der Haut exprimiert ist. Beide bisher bekannten Cannabinoidrezeptoren wurden auf kutanen sensorischen Nervenfasern und Mastzellen nachgewiesen [23]. Werden sie aktiviert, kommt es zu einer klinisch relevanten Unterdrückung von Pruritus, Brennschmerz und Mastzelldegranulation [24]. Auch wenn die genauen molekularen Mechanismen und weitere beteiligte Zielstrukturen dieser Wirkung noch diskutiert werden, wurde bereits in verschiedenen klinischen Applikationen die Wirksamkeit einer Creme-Zubereitung mit N-Palmitoylethanolmin bei juckenden Dermatosen belegt. So wurden in einer groß angelegten klinischen Untersuchung mit über 2000 Patienten inklusive über 900 Kindern bis 12 Jahre die symptomlindernden Effekte bei subakuten und chronischen Stadien der atopischen Dermatitis gezeigt [25]. Des Weiteren liegen Erfahrungen mit diesem Endocannabinoid in kleineren Patientenkollektiven bei der Behandlung verschiedener Pruritusformen vor wie dem renalen Pruritus, dem aquagenen Pruritus, dem brachioradialen Pruritus sowie der Prurigo nodularis [26]. Bislang kann festgestellt werden, dass topische Cannabinoidagonisten in der vorhandenen Formulierung eine effektive antipruritische Therapie mit guter Verträglichkeit bei verschiedenen juckenden Dermatosen insbesondere der AD darstellt und bei Kindern gut einsetzbar ist.

\section{Topische Therapie \\ $\nabla$}

Ergänzend zu den genannten Möglichkeiten einer topischen Therapie können bei akut verstärktem Pruritus weitere topische Präparate eingesetzt werden, die schnell für einen kurzen Zeitraum Pruritus lindern ( Tab. 1). Substanzen wie Polidoca-
Tab. 1 Topische antipruritische Therapiemöglichkeiten bei Kindern

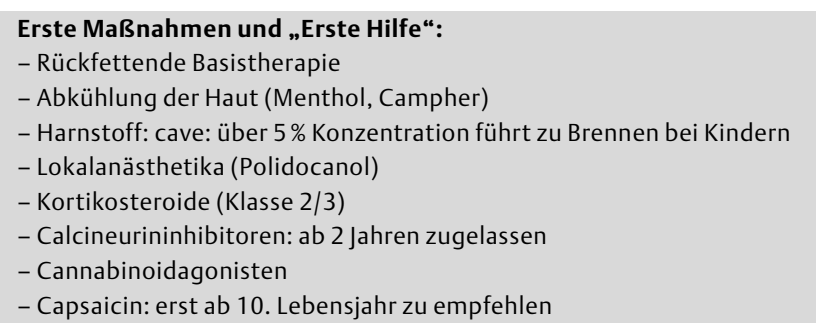

nol (syn: Thesit), Menthol, Campher oder Gerbstoffen können auch bei Kindern eingesetzt werden. Harnstoff kann versuchsweise ebenfalls angewandt werden, kann aber in Konzentrationen ab 5\% zu einem Brennen führen. Wichtig ist auch die Beibehaltung einer Basistherapie, um eine weitere Schädigung der Hautbarriere zu verhindern und einer Trockenheit der Haut vorzubeugen. Beides sind Faktoren, die Pruritus fördern können $[2,5,27]$.

\section{Periphere und zentrale Sensibilisierung $\nabla$}

Durch die Entzündung, die sich bei der AD in der Haut abspielt, gelangen verschiedene Mediatoren mit den Entzündungszellen an die Nervenfasern. Zum Beispiel können eosinophile Granulozyten direkt an Nervenfasern binden; Granulaproteine wie das Eosinophil-derived neurotoxin (EDN) stimulieren Nervenfasern und können Pruritus auslösen. Neurotrophine wie Nerve Growth Factor (NGF), Brain-derived Neurotrophic Factor (BDNF) und Neurotrophin-3 (NT3) führen zu einer Nervenfaserproliferation und peripheren Sensibilisierung [28]. Insbesondere NGF beeinflusst die Neurorezeptoren und bewirkt eine Herabsetzung der Reizschwelle des Histaminrezeptors oder auch des Capsaicinrezeptors (TRPV1) [29]. Durch eine Capsaicintherapie gelingt die Durchbrechung der peripheren Sensibilisierung.

Auch im zentralen Nervensystem (ZNS) kann eine Sensibilisierung durch die ständige Aktivierung der Afferenzen auftreten. So wurde gezeigt, dass kutane Schmerzreize im ZNS als Jucken empfunden werden. Dies erklärt die klinische Beobachtung, dass Patienten durch Kratzen vermehrt Pruritus angeben [30]. Eine Unterbrechung der zentralen Sensibilisierung gelingt nur mit einer systemischen Therapie, z.B. mit Gabapentin oder Naltrexon. Diese Substanzen sind jedoch bei Kindern nicht einsetzbar. Wichtig ist daher eine frühe Intervention und Prävention chronischer Mechanismen bei Kindern. Dies gelingt in erster Linie mit den genannten topischen Therapien einschließlich topischer Steroide; systemische Kortikosteroide (Prednisolon, alternativ Prednison), Immunsuppressiva wie Cyclosporin A (nicht zugelassen, in Studien ab 2. Lebensjahr eingesetzt) oder UV-Therapien (in Ausnahmen ab 10. Lebensjahr) sind in Einzelfällen ebenfalls zielführend ( $\bullet$ Tab. 2) [5,27].

Tab. 2 Systemische antipruritische Therapien

Antihistaminika: je nach Präparat ab 1 bis 12 Jahren zugelassen:

z. B. Cetirizin 1. LJ, Desloratadin 2. LJ

Kortikosteroide: Prednisolon (alternativ Prednison)

Cyclosporin A: nicht zugelassen, in Studien ab 2. LJ eingesetzt

UV-Therapie: in Ausnahmen ab 10. LJ 
Abstract

\section{Pruritus in Atopic Dermatitis: Mechanisms and Therapy}

The incidence of atopic dermatitis in children increased during the past years. Chronic pruritus accompanies the disease; a symptom which is usually difficult to treat by mainly topical therapies in children. Conventional therapies like topical antihistamines are mostly ineffective and lead to prolongation of the symptom. Thus pruritus tends to further chronification and secondary scratching lesions develop along with worsening of the quality of life. Up-to-date, new antipruritic substances are available, which interfere with different pruritogenic mechanisms and which can be applied also in children. Substances which block the cutaneous pruritus induction or transmission to the central nervous system are used to combat pruritus. Important is also an interruption of peripheral sensitization processes underlying chronic pruritus. Unfortunately, to date no approved or certified substances are available for children which interrupt the transmission of pruritus to the central nervous system or central sensitization processes. It is therefore most important to treat children with pruritus in atopic dermatitis as early as possible in order to prevent sensitization and chronification.

\section{Literatur}

1 Mohrenschlager M, Ring J. Atopic eczema. Curr Allergy Asthma Rep 2006; 6: $445-447$

2 Weisshaar E, Seeliger S, Diepgen TL, Luger TA, Ständer S. Pruritus im Kindesalter - eine diagnostische und therapeutische Herausforderung. Hautarzt 2004; 55: 855-868

3 Ständer S, Schmelz M. Chronic itch and pain-similarities and differences. Eur J Pain 2006; 10: 473 - 478

4 Ikoma A, Steinhoff M, Stander S, Yosipovitch G, Schmelz M. The neurobiology of itch. Nat Rev Neurosci 2006; 7: 535-547

5 Ständer S. Rationelle symptomatische Therapie bei chronischem Pruritus. Hautarzt 2006; 57: $403-410$

6 Hsieh ST, Lin WM. Modulation of keratinocyte proliferation by skin innervation. J Invest Dermatol 1999; 113: 579-586

7 Weidner C, Klede M, Rukwied R, Lischetzki G, Neisius U, Skov PS, Petersen $L J$, Schmelz M. Acute effects of substance P and calcitonin gene-related peptide in human skin - a microdialysis study. J Invest Dermatol 2000; 115: 1015 - 1020

8 Tobin D, Nabarro G, de la Faille HB, van Vloten WA, van der Putte SCJ, Schuurman $\mathrm{HJ}$. Increased number of immunoreactive nerve fibers in atopic dermatitis. J Allergy Clin Immunol 1992; 90: 613-622

9 Urashima R, Mihara M. Cutaneous nerves in atopic dermatitis. A histological, immunohistochemical and electron microscopic study. Virchows Arch 1998; 432: $363-370$

10 Pincelli C, Fantini F, Massimi P, Girolomoni G, Seidenari S, Giannetti A. Neuropeptides in skin from patients with atopic dermatitis: an immunohistochemical study. Br J Dermatol 1990; 122: 745 - 750
11 Heyer G, Hornstein OP, Handwerker HO. Skin reactions and itch sensation induced by epicutaneous histamine application in atopic dermatitis and controls. J Invest Dermatol 1989; 93: 492 - 496

12 Klein PA, Clark RA. An evidence-based review of the efficacy of antihistamines in relieving pruritus in atopic dermatitis. Arch Dermatol 1999; 135: 1522 - 1525

13 Rukwied R, Lischetzki G, McGlone F, Heyer G, Schmelz M. Mast cell mediators other than histamine induce pruritus in atopic dermatitis patients: a dermal microdialysis study. Br J Dermatol 2000; 142: 1114 1120

14 Steinhoff M, Vergnolle N, Young SH et al. Agonists of proteinase-activated receptor 2 induce inflammation by a neurogenic mechanism. Nat Med 2000; 6: 151 - 158

15 Steinhoff M, Neisius U, Ikoma A, Fartasch M, Heyer G, Skov PS et al. Proteinase-activated receptor- 2 mediates itch: a novel pathway for pruritus in human skin. J Neurosci 2003; 23: 6176-6180

16 Caterina MJ, Schumacher MA, Tominaga M, Rosen TA, Levine JD, Julius D. The capsaicin receptor: a heat-activated ion channel in the pain pathway. Nature 1997; 389: $816-824$

17 Ständer S, Luger T, Metze D. Treatment of prurigo nodularis with topical capsaicin. J Am Acad Dermatol 2001; 44: 471-478

18 Senba E, Katanosaka K, Yajima H, Mizumura K. The immunosuppressant FK506 activates capsaicin- and bradykinin-sensitive DRG neurons and cutaneous C-fibers. Neurosci Res 2004; 50: 257- 262

19 Ständer S, Ständer H, Seeliger S, Luger TA, Steinhoff M. Topical pimecrolimus (SDZ ASM 981) and tacrolimus (FK 506) transiently induces neuropeptide release and mast cell degranulation in murine skin. $\mathrm{Br} \mathrm{J}$ Dermatol 2007; in press:

20 Edmonds EV, Riaz SN, Francis N, Bunker CB. Nodular prurigo responding to topical tacrolimus. Br J Dermatol 2004; 150: 1216 - 1217

21 Ständer S, Luger TA. Antipruritische Wirkung von Pimecrolimus und Tacrolimus. Hautarzt 2003; 54: 413-417

22 Ständer S, Schürmeyer-Horst F, Luger TA, Weisshaar E. Treatment of pruritic diseases with topical calcineurin inhibitors. Therapeut Clin Risk Management 2006; 2: 213-218

23 Ständer S, Schmelz M, Metze D, Luger T, Rukwied R. Distribution of cannabinoid receptor 1 (CB1) and 2 (CB2) on sensory nerve fibers and adnexal structures in human skin. J Dermatol Sci 2005; 38: 177- 188

24 Rukwied R, Watkinson A, McGlone F, Dvorak M. Cannabinoid agonists attenuate capsaicin-induced responses in human skin. Pain 2003; 102: $283-288$

25 Eberlein-Koenig B, Eicke C, Reinhardt H-W, Ring J. Atopa Study. Abstr. EADV, 2005

26 Ständer S, Reinhardt HW, Luger TA. Topische Cannabinoid-Agonisten: Eine effektive, neue Möglichkeit zur Behandlung von chronischem Pruritus. Hautarzt 2006; 57: $801-807$

27 Ständer S, Streit M, Darsow U, Niemeier V, Vogelgsang M, Ständer H, Gieler U, Gollnick H, Metze D, Weisshaar E. Leitlinie: Diagnostisches und therapeutisches Vorgehen bei chronischem Pruritus. JDDG 2006; 4: $350-370$

28 Raap U, Ikoma A, Kapp A. Neurophysiologie von Pruritus. Hautarzt 2006; 57: 379- 384

29 Shu X, Medell $L M$. Nerve growth factor acutely sensitizes the response of adult rat sensory neurons to capsaicin. J Neurosci 1998; 18: 8947 8959

30 Ikoma A, Rukwied R, Ständer S, Steinhoff M, Miyachi Y, Schmelz M. Sensitization of histamine-induced itch in lesional skin of atopic dermatitis patients. Arch Dermatol 2003; 139: 1455 - 1458 